\title{
Cyclic di-GMP Increases Catalase Production and Hydrogen Peroxide Tolerance in Vibrio cholerae
}

\author{
Nicolas L. Fernandez, ${ }^{a}$ Christopher M. Waters ${ }^{a}$ \\ aDepartment of Microbiology and Molecular Genetics, Michigan State University, East Lansing, Michigan, USA
}

ABSTRACT Vibrio cholerae is a Gram-negative bacterial pathogen that causes the disease cholera, which affects nearly 1 million people each year. In between outbreaks, $V$. cholerae resides in fresh and salt water environments, where it is able to persist through changes in temperature, oxygen, and salinity. One key characteristic that promotes environmental persistence of $V$. cholerae is the ability to form multicellular communities, called biofilms, that often adhere to biotic and abiotic sources. Biofilm formation in $V$. cholerae is positively regulated by the dinucleotide second messenger cyclic dimeric GMP (c-di-GMP). While most research on the c-di-GMP regulon has focused on biofilm formation or motility, we hypothesized that the c-diGMP signaling network encompassed a larger set of effector functions than reported. We found that high intracellular c-di-GMP increased catalase activity $\sim 4$-fold relative to strains with unaltered c-di-GMP. Genetic studies demonstrated that c-diGMP mediated catalase activity was due to increased expression of the catalaseencoding gene katB. Moreover, c-di-GMP mediated regulation of catalase activity and katB expression required the c-di-GMP dependent transcription factors VpsT and VpsR. Lastly, we found that high c-di-GMP increased survival after $\mathrm{H}_{2} \mathrm{O}_{2}$ challenge in a $k a t B-, v p s R$-, and $v p s T$-dependent manner. Our results indicate that antioxidant production is regulated by c-di-GMP uncovering a new node in the growing VpsT and VpsR c-di-GMP signaling network of $V$. cholerae.

IMPORTANCE As a result of infection with $V$. cholerae, patients become dehydrated, leading to death if not properly treated. The aquatic environment is the natural reservoir for $V$. cholerae, where it can survive alterations in temperature, salinity, and oxygen. The second messenger molecule c-di-GMP is an important signal regulating host and aquatic environmental persistence because it controls whether $V$. cholerae will form a biofilm or disperse through flagellar motility. In this work, we demonstrate another function of c-di-GMP in $V$. cholerae biology: promoting tolerance to the reactive oxygen species $\mathrm{H}_{2} \mathrm{O}_{2}$ through the differential regulation of catalase expression. Our results suggest a mechanism where c-di-GMP simultaneously controls biofilm formation and antioxidant production, which could promote persistence in human and marine environments.

KEYWORDS Vibrio cholerae, VpsT, catalase, cyclic di-GMP, reactive oxygen species

\section{T} he Gram-negative bacterium Vibrio cholerae is the human pathogen that causes the diarrheal disease cholera. The most common route to infection is consumption of contaminated food or water, after which $V$. cholerae traverses the stomach and colonizes the small intestines. Cholera patients lose liters of fluid and dissolved ions through toxin-mediated changes to the host intestinal tract, allowing $V$. cholerae to exit the host, reenter a water source, and perpetuate its infectious cycle. In addition to the harsh conditions of the human gastrointestinal tract, $V$. cholerae must adapt to numerous stresses in the aquatic environment. These environmental stresses include temperature
Citation Fernandez NL, Waters CM. 2019 Cyclic di-GMP increases catalase production and hydrogen peroxide tolerance in Vibrio cholerae. Appl Environ Microbiol 85:e01043-19. https://doi.org/10.1128/AEM.01043-19. Editor Maia Kivisaar, University of Tartu Copyright $\odot 2019$ American Society for Microbiology. All Rights Reserved. Address correspondence to Christopher M. Waters, watersc3@msu.edu.

Received 6 May 2019

Accepted 28 June 2019

Accepted manuscript posted online 12 July 2019

Published 29 August 2019 
fluctuations, eukaryotic predation, and exposure to chemical insults such as reactive oxygen species (ROS) (1).

As an aquatic organism, $V$. cholerae is exposed to various concentrations of dissolved oxygen and ROS produced abiotically through photochemical reactions between sunlight and dissolved organic matter in the ocean $(2,3)$. ROS can also be produced biotically through metabolic processes in aerobic environments by phytoplankton, another potential reservoir of $V$. cholerae (4). In response to the multiple routes of exposure to ROS, it is not surprising that $V$. cholerae has multiple ROS defense systems, including two paralogues of the oxidative stress responsive transcription factor OxyR, two catalases, and multiple peroxidases (5-7). Another mechanism to increase tolerance to ROS is the production of surface-adhered communities encased in an exopolysaccharide matrix also known as biofilms.

Many bacterial species, including $V$. cholerae, have increased tolerance to ROS such as hydrogen peroxide $\left(\mathrm{H}_{2} \mathrm{O}_{2}\right)$ when grown in biofilms compared to planktonic counterparts (8-10). Biofilm formation in $V$. cholerae is regulated by the bacterial second messenger molecule cyclic dimeric GMP (c-di-GMP), which is produced by diguanylate cyclase (DGC) enzymes. c-di-GMP alters bacterial physiology by modulating transcription, translation, and/or protein function (11). In V. cholerae, a common mechanism of c-di-GMP signaling is the modulation of gene expression by three c-di-GMP-dependent transcription factors: VpsR, VpsT, and FlrA (12-14). c-di-GMP activates the transcription factors VpsR and VpsT, resulting in increased transcription of genes involved in synthesis of the biofilm matrix component Vibrio polysaccharide (VPS) $(12,13,15)$. In contrast, c-di-GMP acts as an anti-activator of FlrA, which causes decreased expression of genes necessary for flagellar biosynthesis (14). c-di-GMP can also bind to two riboswitches, Vc1 and Vc2. Binding of c-di-GMP to Vc1 functions as an ON-switch to induce production of the adhesin GbpA, while binding of c-di-GMP to Vc2 functions as an OFF-switch to inhibit production of the transcription factor TfoY $(16,17)$. As cells receive signals to disperse from the biofilm, phosphodiesterase (PDE) enzymes, which degrade c-di-GMP, become activated. These PDEs then deplete the intracellular concentration of c-di-GMP, promoting a switch from a sessile biofilm lifestyle to a motile one (reviewed in reference 11).

While VpsR and VpsT were initially discovered as regulators of biofilm production, other c-di-GMP-dependent functions have emerged. For example, c-di-GMP and VpsR transcriptionally regulate genes in the type II secretion operon, as well as $t f o Y$, a gene involved in driving dispersive motility and regulating type VI secretion (17-19). VpsT negatively regulates the expression of genes involved in flagellar biosynthesis; however, the mechanism is not known (13). In addition, Wang and coworkers demonstrated VpsT negatively regulates the transcription of the stationary-phase sigma factor RpoS (20). Recently, we found that c-di-GMP and VpsT induced expression of the DNA repair gene tag to promote survival after alkylation stress (21). These studies demonstrate that c-di-GMP regulation extends beyond biofilm formation and motility in $\mathrm{V}$. cholerae.

In this study, we uncovered an additional role for c-di-GMP: positively regulating catalase activity by increasing transcription of the catalase-encoding gene kat $B$ via a VpsR- and VpsT-dependent mechanism. We further show that c-di-GMP-dependent catalase activity was necessary for survival after exposure to the $\mathrm{ROS} \mathrm{H}_{2} \mathrm{O}_{2}$. Our results expand the regulatory network of c-di-GMP to include antioxidant production, demonstrating that elevated c-di-GMP enhances the oxidative stress response in $\mathrm{V}$. cholerae.

\section{RESULTS}

c-di-GMP positively regulates catalase activity. We have shown that c-di-GMP regulates genes involved in DNA repair and that this regulation increased tolerance to the methylating agent methyl methanesulfonate (21). Thus, we hypothesized c-di-GMP had a role in mitigating other forms of cellular stress besides DNA methylation damage. We chose to test whether c-di-GMP increased $\mathrm{H}_{2} \mathrm{O}_{2}$ tolerance because it is a common ROS produced by aerobic microorganisms as a by-product of cellular respiration, and $\mathrm{H}_{2} \mathrm{O}_{2}$ is found in high concentrations in aquatic environments (reviewed in reference 


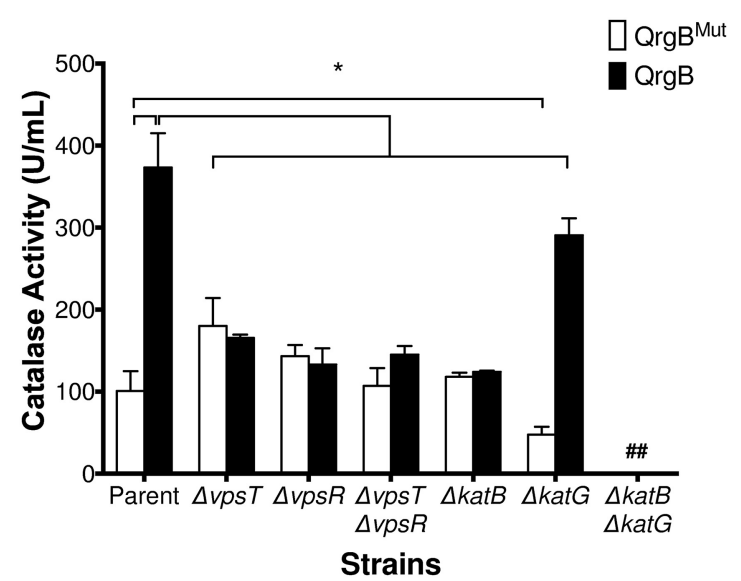

FIG 1 Cyclic di-GMP increases catalase activity. Catalase activity was measured in parent and mutant strains overproducing QrgBMut (open bars) or QrgB (solid bars). Brackets and asterisks (*) indicate differences with a $P$ value of $<0.05$ determined by two-way analysis of variance (ANOVA), followed by Tukey's multiple-comparison test. The data are the average of three biological replicates with error bars indicating the standard deviations. \#\#, activity below the limit of detection.

22). To test the effects of $\mathrm{c}$-di-GMP on $\mathrm{H}_{2} \mathrm{O}_{2}$ tolerance in $V$. cholerae, we expressed the Vibrio harveyi DGC QrgB in a strain unable to form mature biofilms ( $\Delta v p s L$ mutant, designated the parent strain here) which would introduce a confounding variable in our experiments (23). In addition, we chose a heterologous DGC from $V$. harveyi, as opposed to $V$. cholerae, to minimize effects associated with potential protein-protein complexes within the $V$. cholerae c-di-GMP signaling network. As a control, we expressed an inactive allele of $\mathrm{QrgB}$ (QrgBMut) that is unable to synthesize c-di-GMP. Immediately after the addition of $\mathrm{H}_{2} \mathrm{O}_{2}$, both cultures began to produce gas bubbles, which is a phenomenon indicative of catalase activity as $\mathrm{H}_{2} \mathrm{O}_{2}$ is degraded into water and gaseous oxygen. Interestingly, the culture with a higher intracellular c-di-GMP exhibited a larger amount of gas production despite the cultures having similar numbers of bacteria, suggesting that c-di-GMP increased catalase activity. Quantification of catalase activity revealed an approximate 5 -fold increase when comparing strains expressing QrgB to QrgBMut (Fig. 1), indicating c-di-GMP increased catalase activity. Importantly, we have previously demonstrated that the concentration of c-di-GMP generated by QrgB overexpression is similar to that seen naturally in the low-cell-density quorum-sensing state, demonstrating that these results are physiologically relevant (17).

c-di-GMP can directly modulate protein activity or change gene expression through allosteric interactions with c-di-GMP-dependent transcription factors or riboswitches (13-15). The c-di-GMP-dependent transcription factors VpsT and VpsR induce transcription of genes involved in biofilm formation, protein secretion, and DNA repair under high c-di-GMP conditions $(12,13,17,18,21)$. Therefore, we hypothesized that these transcription factors control c-di-GMP-regulated catalase activity. To test this hypothesis, we repeated the catalase assay using $\Delta v p s T, \Delta v p s R$, and $\Delta v p s T \Delta v p s R$ V. cholerae mutants and observed a loss of c-di-GMP-mediated induction of catalase activity, suggesting the increased catalase activity was part of the VpsR/VpsT/c-di-GMP regulatory network (Fig. 1).

V. cholerae has two catalase-encoding genes: katG (VC1560), a bifunctional enzyme with both catalase and peroxidase functions, and katB (VC1585), which only exhibits catalase activity $(7,24)$. Mutants of these enzymes render $V$. cholerae more susceptible to $\mathrm{H}_{2} \mathrm{O}_{2}$ treatment; however, regulation of either katB or kat $G$ by c-di-GMP has not been described (24). We therefore measured c-di-GMP induction of catalase activity in the $\Delta k a t B, \Delta k a t G$, or $\Delta k a t B \Delta k a t G$ mutants. We observed that the $\Delta k a t B$ background did not display c-di-GMP-regulated catalase activity but did possess basal level catalase activity similar to the parent strain expressing QrgB $^{\text {Mut }}$ (Fig. 1). Expression of katB from an 
A

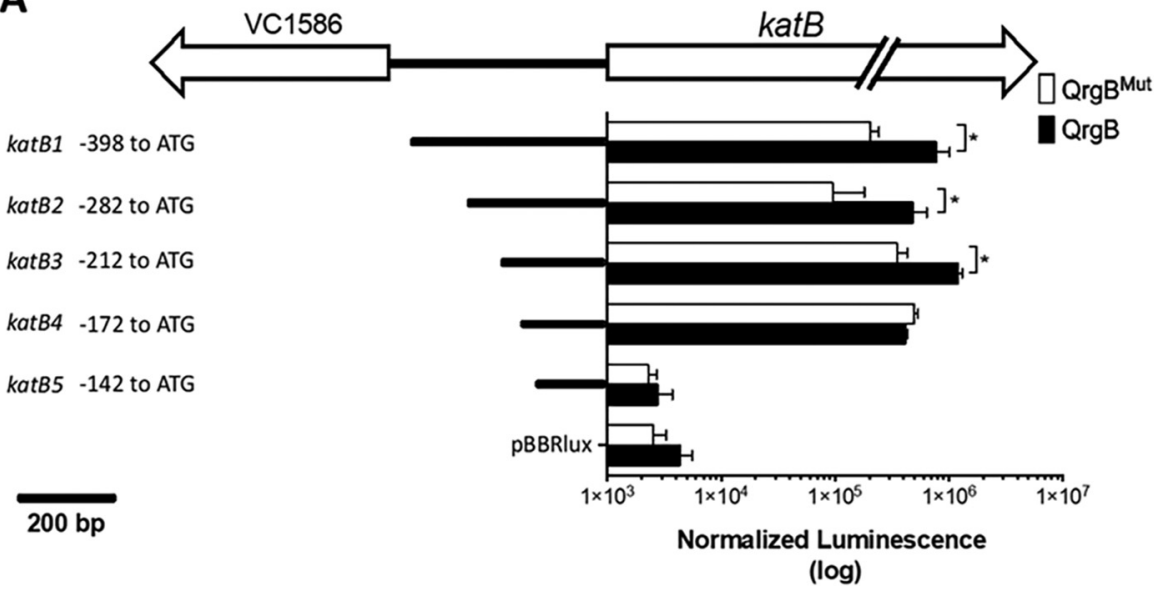

B

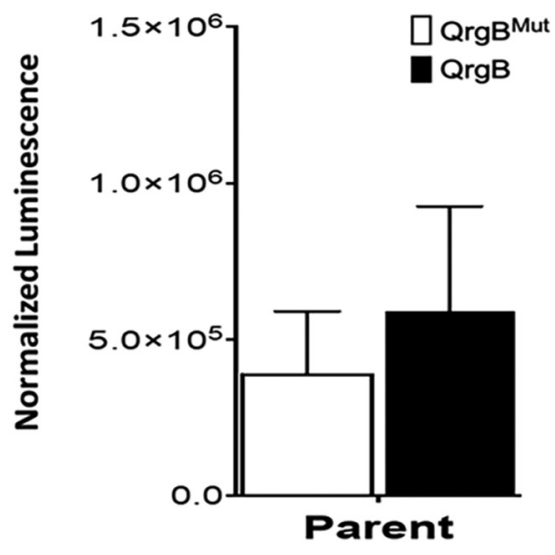

FIG 2 Characterization of katB promoter. (A) katB genetic locus and promoter truncations and reporter activity. The $5^{\prime}$ promoter truncations were constructed and cloned upstream of the luciferase operon. Lines and numbers on the $y$ axis indicate the length of the promoter relative to the ATG start codon for each promoter construct. Normalized luminescence is light production normalized to the $\mathrm{OD}_{600}$ to control for cell number. For each promoter construct, luminescence was measured while overproducing QrgBMut (open bars) or QrgB (solid bars). An empty pBBRlux vector control was included in each experiment. Brackets and asterisks ( $\left.{ }^{*}\right)$ indicate differences with a $P$ value of $<0.05$ determined by two-way ANOVA, followed by Tukey's multiple-comparison test. The data are the average of three biological replicates with error bars indicating the standard deviations. (B) katG expression is unresponsive to changes in c-di-GMP. katG expression was measured in the parent strain while overproducing QrgBMut (open bars) or QrgB (solid bars). Bars represent averages of three biological replicates, with error bars indicating the standard deviations.

arabinose-inducible, multicopy plasmid in the $\Delta k a t B$ background complemented catalase activity regardless of the levels of c-di-GMP (see Fig. S2 in the supplemental material). Strains lacking katG were still able to induce catalase activity by c-di-GMP; however, the level of catalase activity induced by c-di-GMP was approximately $20 \%$ lower than that of the parent strain (Fig. 1). In addition, the catalase activity in the $\Delta$ katG strain expressing QrgB $^{\text {Mut }}$ was $\sim 2$-fold lower than that of the parent strain under the same conditions (Fig. 1). Strains lacking both katG and katB did not have measurable catalase activity under these conditions, which is expected because these are the only annotated genes encoding catalase activity in the El Tor V. cholerae reference genome N16961 (7) (Fig. 1).

Characterization of the katB promoter and regulation of katB expression. Our results suggest $k a t B$, but not $k a t G$, is positively regulated by c-di-GMP at the transcriptional level. We addressed this hypothesis by measuring katB mRNA from cultures inducing QrgB and QrgBMut using quantitative real-time PCR (qRT-PCR). We found that katB expression increased $\sim 12$-fold in QrgB-expressing strains relative to QrgBMut_ expressing strains (see Fig. $\mathrm{S} 1$ in the supplemental material).

We next were interested in the promoter architecture of the region upstream of katB driving expression and hypothesized that specific regions in the kat $B$ promoter were necessary for c-di-GMP-mediated transcriptional control. We tested this hypothesis by measuring luciferase activity from a series of katB transcriptional reporters that have been truncated at the $5^{\prime}$ end in the presence of QrgB or QrgBMut expression (Fig. 2A). In the full-length promoter construct katB1, expression of QrgB induced katB expression 5-fold compared to strains overproducing QrgBMut (Fig. 2A). Inclusion of 212 bp upstream of katB was sufficient to maintain c-di-GMP induction (Fig. 2A, katB2 and katB3). However, if the promoter was truncated to include $172 \mathrm{bp}$ upstream of katB (katB4), c-di-GMP-dependent induction of $k a t B$ was abrogated. This result suggested the necessary cis-acting sequences for c-di-GMP-mediated activation of katB expression are found between -172 and -212 bp upstream of the katB relative to the ATG start codon (Fig. 2A). Deleting $30 \mathrm{bp}$ from katB4 resulted in expression levels similar to that of the promoterless vector control regardless of the c-di-GMP concentrations, suggesting components necessary for the basal expression of katB are between -172 and -142 relative to the ATG start codon (Fig. $2 \mathrm{~A}$ ). We also measured the effect of c-di-GMP 
A

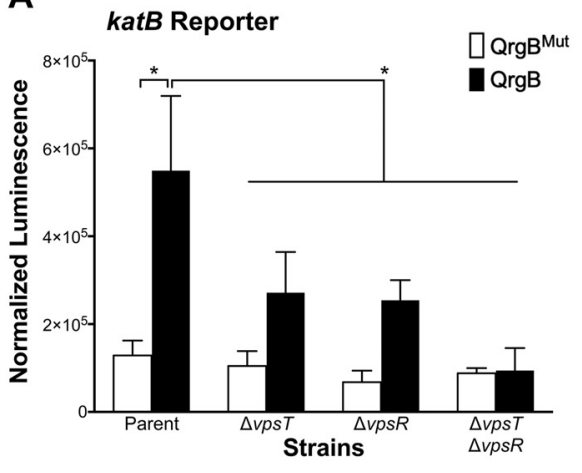

B

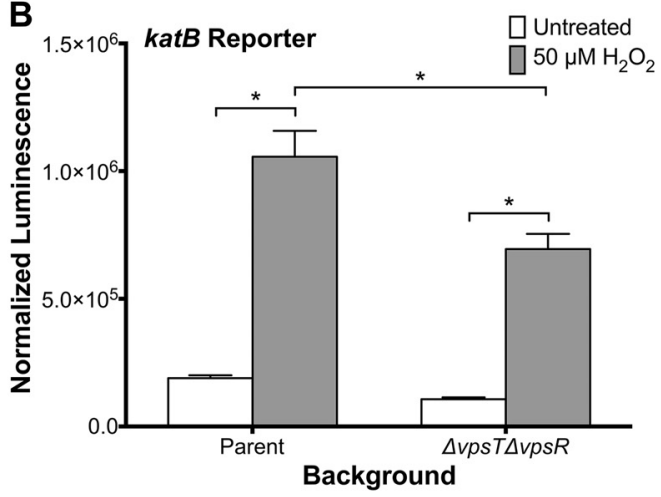

FIG 3 vpsT and $v p s R$ are Necessary for c-di-GMP mediated expression of katB but dispensable for $\mathrm{H}_{2} \mathrm{O}_{2}$ induction. (A) katB expression was measured in parent, $\Delta v p s T, \Delta v p s R$, and $\Delta v p s T \Delta v p s R$ backgrounds while overproducing QrgBMut (white bars) or QrgB (black bars). Brackets and asterisks $\left(^{*}\right.$ ) indicate differences with a $P$ value of $<0.05$ determined by two-way ANOVA, followed by Tukey's multiple-comparison test. The data are the average of three biological replicates with error bars indicating the standard deviations. (B) katB4 expression was measured in parent and $\Delta v p s T \Delta v p s R$ backgrounds 30 min after the addition of $50 \mu \mathrm{M} \mathrm{H}_{2} \mathrm{O}_{2}$ or PBS control (untreated). The data are averages of three biological replicates, and error bars indicate the standard deviations.

on a katG-luciferase transcriptional fusion and did not observe significant c-di-GMPdependent changes in expression, in agreement with our initial hypothesis (Fig. 2B).

VpsT and VpsR are necessary for the c-di-GMP-dependent induction of catalase activity, suggesting that these transcription factors activate transcription of katB at high intracellular concentrations of c-di-GMP (Fig. 1). Thus, we hypothesized that c-di-GMPmediated induction of kat $B$ would be lost in strains lacking $v p s T, v p s R$, or both vpsT and $v p s R$. We tested this hypothesis by measuring katB2 reporter activity under different c-di-GMP conditions in the parent, single-knockout, and double-knockout strains. We found that katB2 expression increased 5-fold in the parent background. katB expression increased 2.5- to 3-fold in the $\Delta v p s T$ and $\Delta v p s R$ backgrounds, but the differences in expression between QrgBmut and QrgB were not statistically significant (Fig. 3A). It was only in the double-mutant $\Delta v p s T \Delta v p s R$ background that c-di-GMP-mediated katB expression was completely lost (Fig. 3A). These data suggest that both VpsR and VpsT are needed for full induction by c-di-GMP and that individually they may be able to change expression of the katB reporter. However, if induction of katB occurs in the single $\Delta v p s T$ and $\Delta v p s R$ mutants, it is not sufficient to alter catalase activity (Fig. 1).

$k a t B$ expression is upregulated in response to hydrogen peroxide in $V$. cholerae through the transcriptional activator $\operatorname{OxyR}(6,24)$. To determine whether the same region necessary for c-di-GMP-mediated regulation of katB was necessary for $\mathrm{H}_{2} \mathrm{O}_{2}$ induction of katB, we measured the ability of $\mathrm{H}_{2} \mathrm{O}_{2}$ to induce katB4, the promoter region that no longer responded to c-di-GMP (Fig. 2A). We found that $50 \mu \mathrm{M} \mathrm{H}_{2} \mathrm{O}_{2}$ induced katB4 expression $\sim 6$-fold in the parent background (Fig. 3B). Next, to determine whether VpsT and VpsR contributed to the $\mathrm{H}_{2} \mathrm{O}_{2}$ inducible response of the katB4 promoter, we repeated the assay in a $\triangle v p s T \Delta v p s R$ background. In this background, $\mathrm{H}_{2} \mathrm{O}_{2}$ increased katB4 expression to the same extent as the parent; however, the basal and induced expression level was 1.5-fold lower compared to the parent (Fig. 3B).

In Mycobacterium smegmatis, $\mathrm{H}_{2} \mathrm{O}_{2}$ can act as a first messenger to promote c-di-GMP synthesis (25). Whether $\mathrm{H}_{2} \mathrm{O}_{2}$ acts as a first messenger to modulate c-di-GMP in $\mathrm{V}$. cholerae has not been demonstrated. To test the hypothesis that $\mathrm{H}_{2} \mathrm{O}_{2}$ acts as a first messenger, we measured intracellular c-di-GMP in control and $\mathrm{H}_{2} \mathrm{O}_{2}$-treated cultures and found no differences in intracellular c-di-GMP (see Fig. S3 in the supplemental material). Together, these results indicate that katB transcription is induced by c-di-GMP and the oxidative stress response through distinct regulatory mechanisms and demonstrate that $\mathrm{H}_{2} \mathrm{O}_{2}$ does not alter global levels of intracellular c-di-GMP.

VpsT induces kat $B$ expression in a heterologous host and binds to the kat $B$ promoter in vitro. To test whether VpsT or VpsR directly regulates katB, we used 


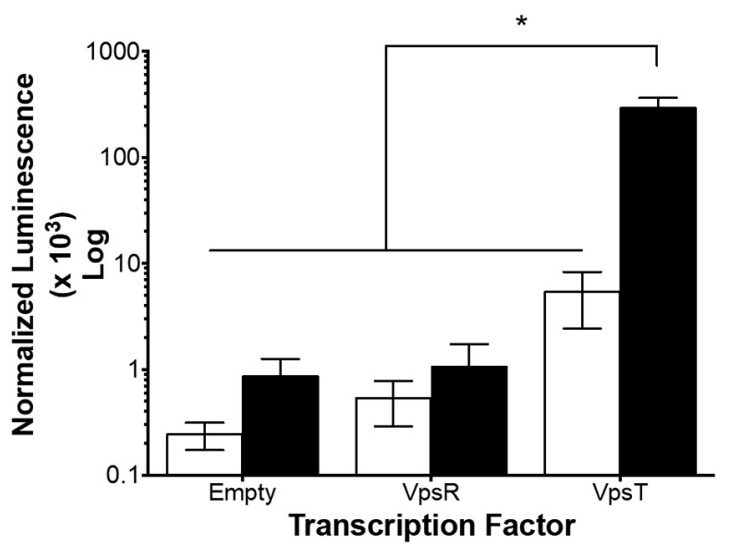

FIG 4 VpsT and c-di-GMP induce katB expression in a heterologous host. QrgBMut (open bars) or QrgB (solid bars) was overproduced, along with either an empty vector, VpsR, or VpsT in DH10b E. coli harboring the katB3 promoter fused upstream of the luciferase operon. The data are average luminescences between strains overproducing QrgB and QrgBMut from three biological replicates, and error bars indicate the standard deviations. Brackets and asterisks $\left(^{*}\right)$ indicate statistically significant differences determined by two-way ANOVA, followed by Tukey's multiple-comparison testing.

Escherichia coli as a heterologous host to measure katB expression at high versus low concentrations of c-di-GMP in the presence of either VpsR or VpsT. We reasoned that the genetic dissimilarity between $V$. cholerae and E. coli would allow us to isolate the effects of VpsR and VpsT directly on the katB promoter without the regulatory feedback for these transcription factors $(23,26,27)$. Expression of QrgB with an empty vector increased katB expression in $E$. coli $\sim 2.5$-fold compared to expression of QrgBMut through an unknown mechanism (Fig. 4). Similarly, coexpression of VpsR with QrgB resulted in approximately the same fold change (2-fold) as the empty vector, indicating that VpsR is not sufficient to induce katB expression when expressed in $E$. coli along with increased c-di-GMP (Fig. 4). Interestingly, expression of VpsT with QrgBMut increased katB3 expression 20-fold compared to the empty vector control. Although this comparison had a $P$ value of 0.8 , the large difference between averages suggests VpsT can contribute to modulating gene expression under low-c-di-GMP conditions (Fig. 4). The largest difference in katB3 expression was observed when VpsT was expressed with QrgB, resulting in a 344-fold induction compared to the empty vector (Fig. 4). Together, these data suggest VpsT is a key regulator of katB expression and that transcriptional activation is increased when c-di-GMP is present.

As a transcription factor, VpsT binds to DNA in the presence of c-di-GMP to modulate gene expression $(13,20,21,27)$. Since VpsT was necessary for c-di-GMPmediated $k a t B$ expression and was able to induce katB expression when expressed in a heterologous host, we hypothesized VpsT directly interacted with the katB2 promoter in a c-di-GMP-dependent manner. Thus, we purified C-terminal His-tagged VpsT and measured its ability to bind to the katB2 promoter in vitro. VpsT only partially shifted the katB2 probe at the highest concentration tested $(600 \mathrm{nM})$ in the absence of c-di-GMP (Fig. 5, lane 5). However, with the addition of $50 \mu \mathrm{M} \mathrm{c-di-GMP,} \mathrm{VpsT} \mathrm{was} \mathrm{able}$ to decrease the intensity of the unshifted band at $150 \mathrm{nM}$ and completely shift the probe at $300 \mathrm{nM}$ (Fig. 5, lanes 10 to 12). The addition of an unlabeled, 20-bp oligonucleotide composed of a VpsT binding site found in the vpsL promoter at a 100-fold molar excess was able to outcompete VpsT binding to the labeled katB2 probe in the presence or absence of c-di-GMP (Fig. 5, lanes 7 and 14) (27). When the unlabeled competitor had transversion mutations introduced into the palindromic region, it was no longer able to abrogate the VpsT-katB2 band migration (Fig. 5, lanes 6 and 13) (27). Together, the in vivo and in vitro data suggest that VpsT directly interacts with the katB promoter to induce expression under high-c-di-GMP conditions.

c-di-GMP-mediated HOOH survival is dependent on catalase. Since c-di-GMP increased katB expression and catalase activity, we hypothesized that high levels of 


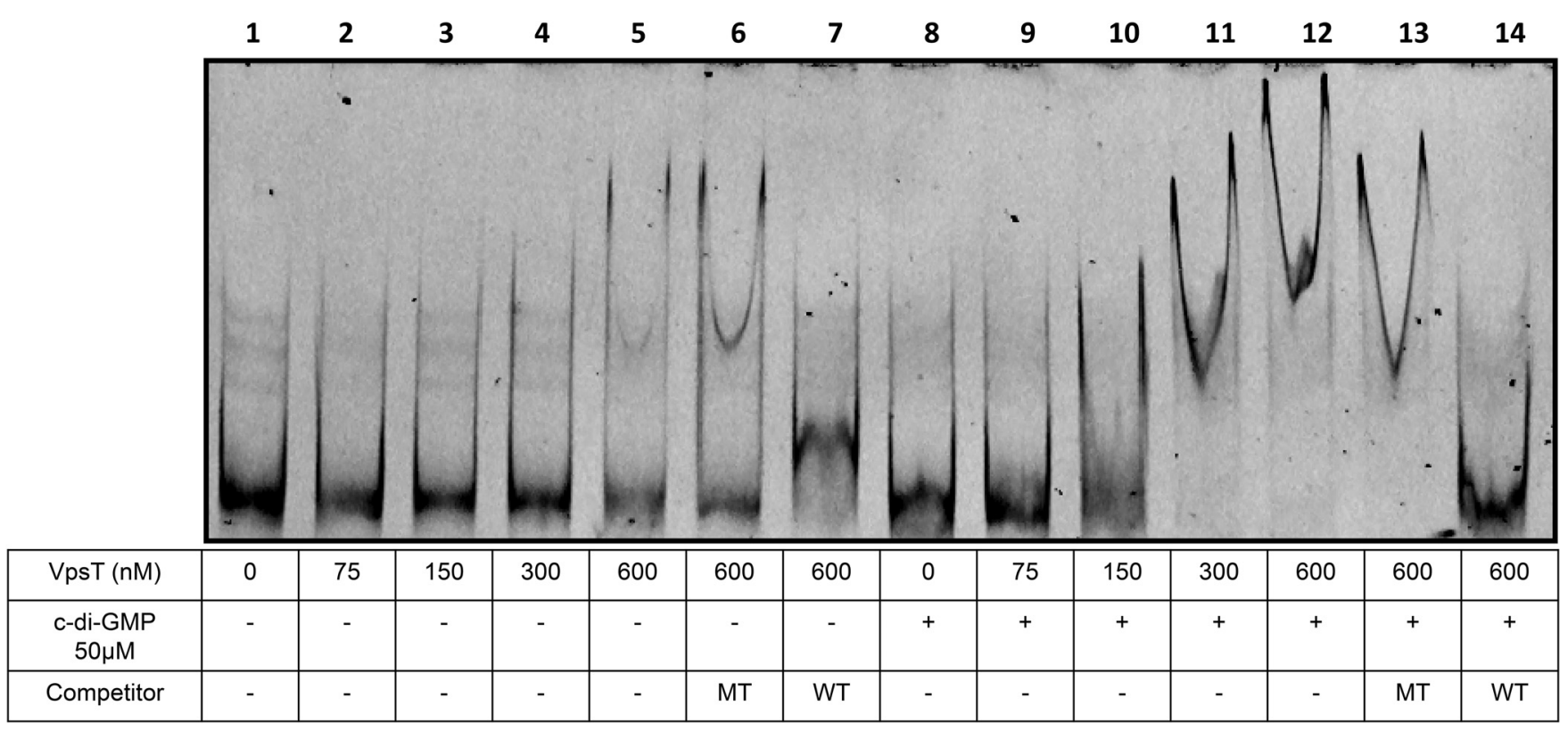

FIG 5 c-di-GMP enhances VpsT interaction with the katB promoter in vitro. Increasing concentrations of VpsT-HIS were incubated with a FAM-labeled probe corresponding to the katB4 promoter (lanes 1 to 5). Unlabeled mutant (MT) or wild-type (WT) VpsT binding site competitor was added at a 100X molar excess relative to the labeled probe in reactions with $600 \mathrm{nM}$ VpsT-His (lanes 6 and 7). Lanes 8 to 14 represent the same reaction conditions as lanes 1 to 7 except that $50 \mu \mathrm{M}$ c-di-GMP was added to the binding reactions (indicated by the "+" sign).

intracellular c-di-GMP would provide a survival advantage during $\mathrm{H}_{2} \mathrm{O}_{2}$ stress. We tested this hypothesis by measuring survival after $\mathrm{H}_{2} \mathrm{O}_{2}$ treatment in $\mathrm{V}$. cholerae backgrounds $(\Delta v p s L)$ unable to make mature biofilms to specifically test whether the transcription regulation of katB by c-di-GMP was responsible for any observed protection as opposed to matrix production or the formation of multicellular biofilms $(8,10$, $28,29)$. V. cholerae strains expressing $\mathrm{QrgB}^{\mathrm{Mut}}$ or $\mathrm{QrgB}$ were challenged with $\mathrm{H}_{2} \mathrm{O}_{2}$, and the gross culture viability (i.e., the optical density at $600 \mathrm{~nm}\left[\mathrm{OD}_{600}\right]$ ) was monitored for $3 \mathrm{~h}$. We found that in the parent strain the expression of QrgB, but not QrgBMut, led to significant protection from $\mathrm{H}_{2} \mathrm{O}_{2}$ stress (Fig. $6 \mathrm{~A}$ ). To test whether this production was dependent on c-di-GMP-mediated catalase activity, we measured $\mathrm{H}_{2} \mathrm{O}_{2}$ survival in the $\Delta k a t B, \Delta k a t G$, and $\Delta k a t B \Delta k a t G$ mutants. Consistent with our catalase activity results, deletion of $k a t B$ in either the $\triangle k a t B$ and $\triangle k a t B \Delta k a t G$ mutants resulted in no c-di-GMPmediated $\mathrm{H}_{2} \mathrm{O}_{2}$ survival (Fig. $6 \mathrm{E}$ and $\mathrm{G}$ ), whereas the survival of the $\Delta k a t G$ mutant during $\mathrm{H}_{2} \mathrm{O}_{2}$ treatment resembled that of the parent strain (Fig. 6F).

Since vpsT and vpsR were necessary for c-di-GMP-dependent induction of katB expression and catalase activity (Fig. 1 and 4), we hypothesized that deletion mutations of these transcription factors would decouple c-di-GMP signaling from survival during $\mathrm{H}_{2} \mathrm{O}_{2}$ treatment. Indeed, strains lacking $v p s T$, vpsR, or both $v p s T$ and $v p s R$ lost c-di-GMPmediated survival during $\mathrm{H}_{2} \mathrm{O}_{2}$ treatment (Fig. $6 \mathrm{~B}$ to D). Taken together, these data suggest that c-di-GMP increases katB expression and KatB catalase activity through the c-di-GMP-dependent transcription factors vps $T$ and $v p s R$, resulting in increased survival during $\mathrm{H}_{2} \mathrm{O}_{2}$ treatment (Fig. 7).

\section{DISCUSSION}

We sought here to determine whether providing resistance to ROS is a c-di-GMPregulated phenotype in $V$. cholerae. Using a plasmid-based system to modulate intracellular c-di-GMP, we observed increased gas production after the addition of $\mathrm{H}_{2} \mathrm{O}_{2}$, suggesting that c-di-GMP positively influenced catalase activity. We determined that $k a t B$ was responsible for $c$-di-GMP-regulated catalase activity and found that katB transcription was increased 5 -fold in the parent background but was lost in strains lacking the c-di-GMP-dependent transcription factors VpsT and VpsR. Measuring katB 


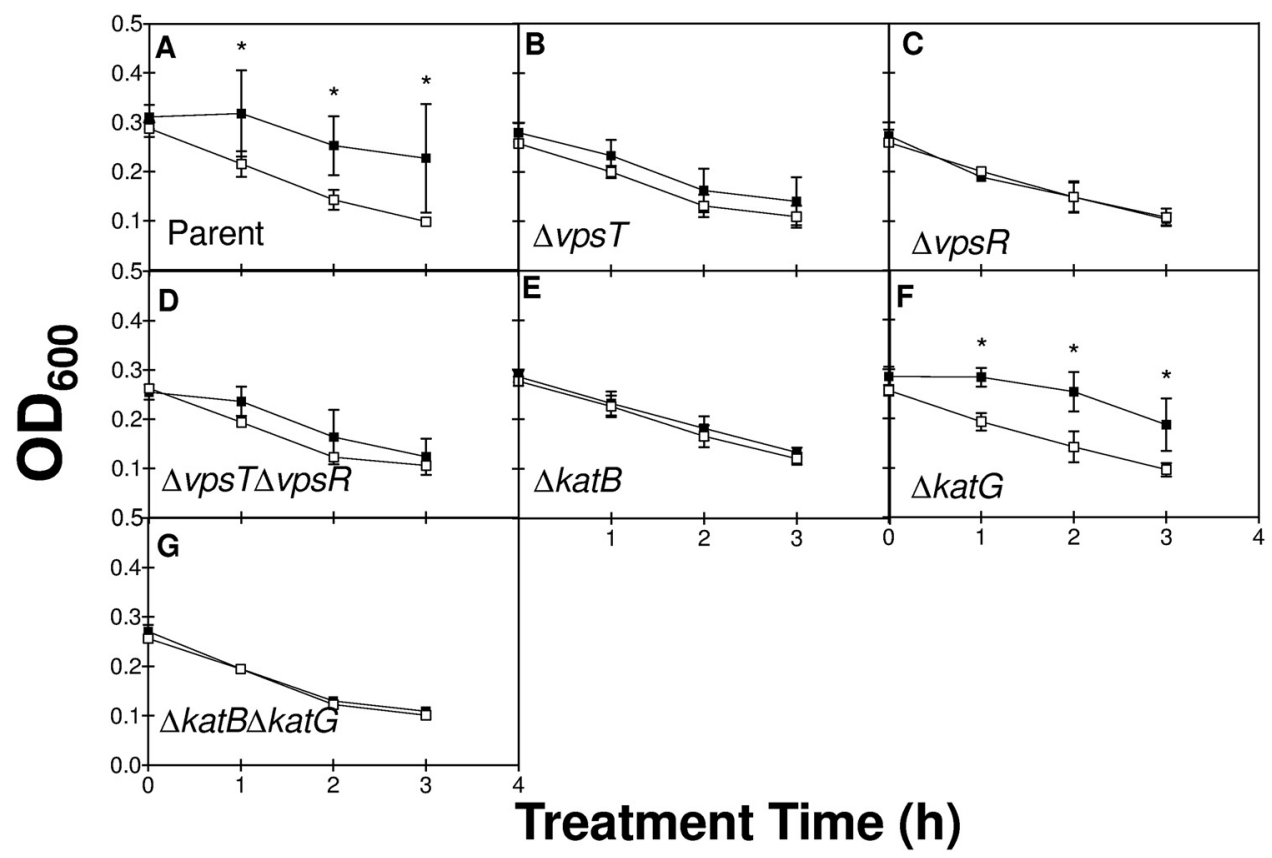

FIG 6 c-di-GMP enhances survival after $\mathrm{H}_{2} \mathrm{O}_{2}$ treatment. QrgBMut (open squares) or QrgB (solid squares) were induced with $100 \mu \mathrm{M}$ IPTG in parent (A), $\Delta v p s T$ (B), $\Delta v p s R(\mathrm{C}), \Delta v p s T \Delta v p s R(\mathrm{D}), \Delta k a t B(\mathrm{E}), \Delta k a t G(\mathrm{~F})$, and $\Delta k a t B \Delta k a t G$ (G) strains until reaching an $\mathrm{OD}_{600}$ of $0.3 . \mathrm{H}_{2} \mathrm{O}_{2}$ was added to cultures at a final concentration of $12.5 \mathrm{mM}$. Cell death was monitored by measuring $\mathrm{OD}_{600}$ every hour after $\mathrm{H}_{2} \mathrm{O}_{2}$ addition (time 0 ). ${ }^{*}, P<0.05$ compared to the untreated control at that time point as determined by two-way ANOVA, followed by Tukey's multiple-comparison test. Bars indicate averages from three biological replicates, and error bars indicate the standard deviations.

transcription in a heterologous host revealed that VpsT was sufficient to induce katB expression under high-c-di-GMP conditions, and in vitro DNA binding assays demonstrated that VpsT specifically bound to the katB promoter in a c-di-GMP-dependent fashion. Lastly, we showed that c-di-GMP-mediated survival after $\mathrm{H}_{2} \mathrm{O}_{2}$ treatment was dependent on $v p s T$, vpsR, and katB.

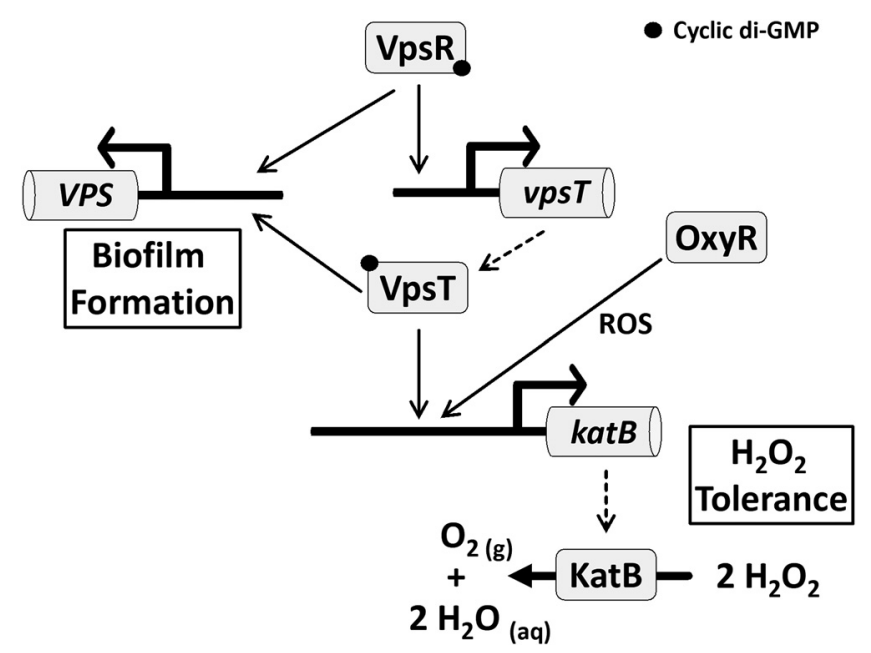

FIG 7 Model for the cyclic di-GMP regulatory network controlling biofilm formation and $\mathrm{H}_{2} \mathrm{O}_{2}$ tolerance. In response to high intracellular levels of cyclic di-GMP, VpsR becomes activated and induces the expression of vpsT. VpsT, in turn, is activated by cyclic di-GMP, and both VpsR and VpsT activate genes in the vps operons to promote biofilm formation. Our data indicate that cyclic di-GMP-activated VpsT also induces the expression of $k a t B$, which increases the catalase activity of the population. The catalase degrades $\mathrm{H}_{2} \mathrm{O}_{2}$ and promotes survival after $\mathrm{H}_{2} \mathrm{O}_{2}$ treatment. Our data also demonstrate that the induction of katB by $\mathrm{H}_{2} \mathrm{O}_{2}$ occurs independently of the cyclic di-GMP signaling network and is likely controlled by OxyR, a transcription factor involved in a broader antioxidant production program. 
In agreement with our findings, other groups have observed an increase in katB expression under certain biofilm-inducing conditions that modulate intracellular c-diGMP, such as incubation with norspermidine (30). In addition, rugose variants of $V$. cholerae were shown to be resistant to $\mathrm{H}_{2} \mathrm{O}_{2}$ in a VPS-independent manner and were later found to have increased expression of $\operatorname{katB}(31,32)$. Transcriptomic data from experiments where c-di-GMP or VpsT was artificially induced in V. cholerae suggested that katB expression was positively regulated by c-di-GMP $(13,33)$.

Interestingly, c-di-GMP and VPsT were shown to downregulate the production of the stationary-phase sigma factor RpoS, in turn decreasing survival to various stressors, including $\mathrm{H}_{2} \mathrm{O}_{2}$, which is in contrast to our results (Fig. 5A) (20). These experiments were done with the El Tor biotype strain C7258, which is part of the serogroup Ogawa, whereas the strain used in the present study (C6076 Str2) is part of the serogroup Inaba. Although there are no studies describing differences in transcriptional regulation between the two serogroups, we hypothesize there may be serogroup-dependent differences in c-di-GMP signaling, which would explain the contrasting results.

Unlike the $\Delta v p s T \Delta v p s R$ double mutant, c-di-GMP was able to induce the expression of the katB reporter in the single vpsT and $v p s R$ deletion backgrounds, although the difference was not statistically significant. To further clarify which transcription factor was the direct effector, we reconstructed the system in E. coli and found that only production of VpsT and c-di-GMP, but not that of VpsR and c-di-GMP, increased the expression of the katB promoter in E. coli (Fig. 4). c-di-GMP-mediated catalase activity and survival in $\mathrm{H}_{2} \mathrm{O}_{2}$ were also lost in both $\Delta v p s T$ and $\Delta v p s R$ backgrounds. Thus, our evidence suggests that phenotypes are primarily controlled by VpsT (Fig. 7). VpsR is required for c-di-GMP-mediated induction of vpsT in V. cholerae; therefore, we conclude that it has an indirect effect on katB transcription (26) (Fig. 7).

Interestingly, we observed that VpsT was able to induce katB expression 20-fold under unaltered c-di-GMP conditions in E. coli (Fig. 4). In addition, VpsT was also able to bind to the katB promoter without exogenously added c-di-GMP, albeit at high protein concentrations (Fig. 5). It is possible these results are a consequence of relatively high VpsT levels driven by the expression plasmid; however, it is also possible that VpsT may have some c-di-GMP-independent effect on gene expression. Indeed, allele variants of VpsT that are unable to bind to c-di-GMP are still able to modulate gene expression (13). If and how VpsT functions in the absence of c-di-GMP is an area that requires more research.

A predicted VpsT binding site $\sim 150$ bp upstream of the ATG start codon of katB was previously reported (34). Our results indicate that even when the VpsT binding site was present in the katB4 transcriptional fusion, induction by c-di-GMP was lost. We note that the predicted VPsT sequence was found in the template strand, while predicted and validated VpsT binding sites in the vps $L$ and vpsA promoters were found in the coding strand $(27,34)$. As a result of this difference, the binding site would position VpsT in different directionalities, i.e., positioned toward or away from the open reading frame. Whether this difference in directionality alters VpsT transcriptional regulation warrants further investigation.

Although our work focuses on $V$. cholerae, the association between c-di-GMP and protection against ROS has been shown in other bacteria. In Listeria monocytogenes, deletion of genes encoding proteins with PDE domains resulted in elevated biofilm production and $\mathrm{H}_{2} \mathrm{O}_{2}$ tolerance (9). However, it is not known whether the $\mathrm{H}_{2} \mathrm{O}_{2}$ tolerance was caused by increased EPS production, antioxidant production, or both. In Mycobacterium smegmatis, a relative of the human pathogen $M$. tuberculosis, $\mathrm{H}_{2} \mathrm{O}_{2}$ stimulates the production of intracellular c-di-GMP, which inactivates the transcriptional repressor HpoR. As a result, ROS defense genes are upregulated and increase $\mathrm{H}_{2} \mathrm{O}_{2}$ tolerance (25). This differs from our work in two ways: (i) $\mathrm{H}_{2} \mathrm{O}_{2}$ does not act as a first messenger that stimulates c-di-GMP activity in V. cholerae (see Fig. S3) and (ii) VpsT and c-di-GMP regulate a wide array of genes, whereas HpoR-c-di-GMP has been shown to only regulate expression of the $h p o R$ operon. Despite the differences in regulation, 
TABLE 1 Plasmids used in this study

\begin{tabular}{|c|c|c|c|}
\hline Plasmid & Description $^{a}$ & Copy no. & Source or reference \\
\hline pBBRlux & luxABCDE containing promoterless plasmid; $\mathrm{Cam}^{\mathrm{r}}$ & $5-10$ & Lab collection \\
\hline pEVS143 & pTac overexpression vector; $\operatorname{Kan}^{r}$ & 10 & 40 \\
\hline pKAS32 & $\lambda$ pir-dependent allele exchange vector; $\mathrm{Amp}^{\mathrm{r}}$ & $15-20$ & 41 \\
\hline pBRP333 & Control expression vector; Kan ${ }^{r}$ & 10 & 17 \\
\hline pBRP1 & qrgB* (inactive DGC) cloned into expression vector pMMB67Eh; Amp ${ }^{r}$ & $10-12$ & 17 \\
\hline pBRP2 & qrgB (active DGC) cloned into expression vector, pMMB67Eh; Ampr & $10-12$ & 17 \\
\hline pNF031 & katB1 promoter in pBBRlux; Cam ${ }^{r}$ & $5-10$ & This study \\
\hline pNF032 & katB2 promoter in pBBRlux; Cam ${ }^{r}$ & $5-10$ & This study \\
\hline pNF033 & katB3 promoter in pBBRlux; Cam ${ }^{r}$ & $5-10$ & This study \\
\hline pNF036 & katB4 promoter in pBBRlux; Cam ${ }^{r}$ & $5-10$ & This study \\
\hline pNF038 & katB5 promoter in pBBRlux; Cam ${ }^{r}$ & $5-10$ & This study \\
\hline pNF030 & katG promoter in pBBRlux; Camr & $5-10$ & This study \\
\hline pCMW131 & $v p s R$ in pEVS143; Kan ${ }^{r}$ & 10 & 23 \\
\hline pCMW132 & vpsT in pEVS143; Kan ${ }^{r}$ & 10 & 23 \\
\hline pNF011 & vpsT in pET28b (C-terminal His tag); Kan ${ }^{r}$ & $15-20$ & 21 \\
\hline pNF057 & pHERD20T ${ }_{p}$ BAD expression vector, with Cam ${ }^{r}$ replacing Amp $^{r}$ & $10-15^{*}$ & This study \\
\hline pNF067 & katB in pNF057; Cam ${ }^{r}$ & $10-15^{*}$ & This study \\
\hline pNF068 & katG in pNF057; Camr & $10-15^{*}$ & This study \\
\hline pNF045 & Deletion construct for katB in pKAS32; Amp ${ }^{r}$ & $15-20$ & This study \\
\hline pNF048 & Deletion construct for katG in pKAS32; Ampr & $15-20$ & This study \\
\hline
\end{tabular}

${ }^{a}$ Camr ${ }^{r}$, chloramphenicol resistance; Kanr, kanamycin resistance; Ampr ${ }^{r}$, ampicillin resistance. ${ }^{*}$, the copy number of the pRO1600 origin is host dependent.

the connection between c-di-GMP and ROS tolerance is evident in bacteria from diverse phylogenetic backgrounds.

\section{MATERIALS AND METHODS}

DNA manipulations and growth conditions. V. cholerae C6706 Str2 was used as the wild type, and the low-biofilm-forming $\Delta v p s L$ mutant derivative was used as the parent strain $(23,35)$. All vectors were constructed by Gibson Assembly (NEB). Chromosomal deletion strains were constructed using the allele exchange vector pKAS32 digested with Kpnl and Sacl (NEB, High Fidelity). Luciferase reporters were constructed using the luciferase reporter vector pBBRlux digested with BamHI and Spel (NEB) (Table 1). Expression vectors for VpsT and VpsR were constructed by removing the ribosome-binding site, green fluorescent protein, and chloramphenicol acetyltransferase from pEVS143 with BamHI and EcoRI digests (NEB) (23). The VpsT purification vector was constructed elsewhere (21). Expression vectors for katB and katG were constructed as follows. PHERD20T was amplified with primers flanking the ampicillin resistance gene using inverse $P C R$, resulting in a linear PCR fragment lacking the ampicillin resistance gene (36). The chloramphenicol resistance gene from pBBRlux was amplified by PCR, and the two linear fragments were circularized by Gibson Assembly. Plasmids were introduced into $V$. cholerae through

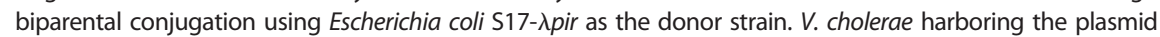
of interest was selected for using polymyxin $B(10 \mathrm{U} / \mathrm{ml})$ with the relevant antibiotic. Antibiotics and reagents were used at the following concentrations unless stated otherwise: ampicillin $(100 \mu \mathrm{g} / \mathrm{ml})$, kanamycin $(100 \mu \mathrm{g} / \mathrm{ml})$, chloramphenicol $(10 \mu \mathrm{g} / \mathrm{ml})$, and $100 \mu \mathrm{M}$ IPTG (isopropyl- $\beta$-D-thiogalactopyranoside). Cultures were grown in lysogeny broth (LB; Acumedia) at $35^{\circ} \mathrm{C}$ and $220 \mathrm{rpm}$ unless otherwise stated (Table 2 shows strains used in this study).

TABLE 2 Strains used in this study

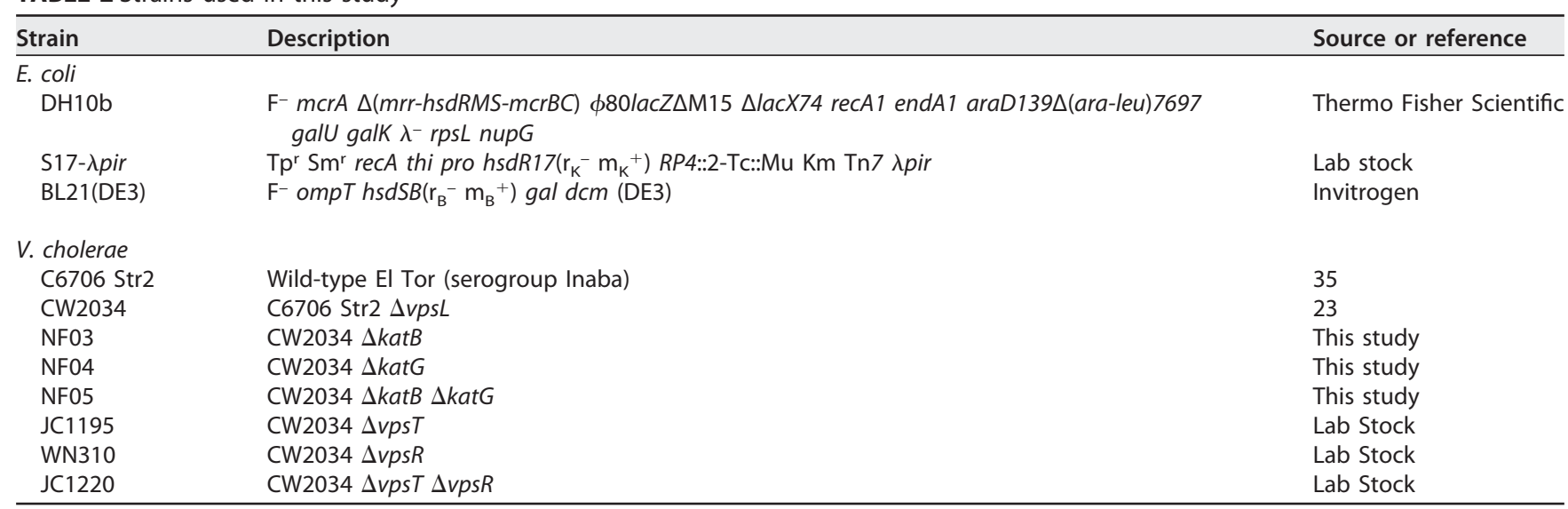


TABLE 3 Oligonucleotides used for qRT-PCR and EMSA experiments

\begin{tabular}{lll}
\hline Expt and description & Sequence $\left(5^{\prime}-3^{\prime}\right)$ & $T_{m}{ }^{a}$ (qRT-PCR only) \\
\hline qRT-PCR & AAATTCATCTTCATGGCAAC & 58 \\
gyrA-For & GCGATGTITCTCACAG & 58 \\
gyrA-Rev & CGTATGCCGATACACAGCTTTA & 64 \\
katB-For & ACGACTGGGTGGTAGTTAATG & 64 \\
EMSA & \\
pBBRLux-FAM-For & \\
pBBRLux-FAM-Rev & FAM-ATITGCGGCCGCAACTAGA \\
WT-VpsT binding site-For & FAM-CCGCGGTGGGGCCGCTCTA \\
WT-VpsT binding site-Rev & AAAGTAAACTAAAGTTATTIT \\
MT-VpsT binding site-For & AAAATAAACTTAGTTACTTT \\
MT-VpsT binding site-Rev & AAAAGGTCTAAAACTAGGTT \\
\hline
\end{tabular}

${ }^{a} T_{m}$, melting temperature.

Catalase assay. Measurement of catalase activity was adapted from an earlier study (37) with the following changes. Overnight cultures were diluted 1:100, the $\mathrm{OD}_{600}$ was measured, and the resulting value was multiplied by the dilution factor of 100 to obtain the actual optical density of the solution. The cell solutions were diluted to a starting $\mathrm{OD}_{600}$ of $0.040 \mathrm{in} 5 \mathrm{ml}$ of $\mathrm{LB}$ in 18 - by 150 -mm borosilicate test tubes supplemented with the necessary antibiotics and IPTG. Cultures were grown at $35^{\circ} \mathrm{C}$ with shaking at $220 \mathrm{rpm}$ until the $\mathrm{OD}_{600}$ reached $\sim 2.0$, moved to $15-\mathrm{ml}$ Falcon tubes (Corning), and pelleted by centrifugation $(4,000 \times g$ for $3 \mathrm{~min})$. Pellets were resuspended in $100 \mu \mathrm{l}$ of sterile $1 \times$ phosphate-buffered saline (PBS) to create a viscous cell solution that was adjusted to an $\mathrm{OD}_{600}$ of 150 in a $100-\mu$ l final volume in Pyrex test tubes ( 13 by $100 \mathrm{~mm}$, borosilicate). Then, $200 \mu \mathrm{l}$ of catalase reaction buffer ( $1 \%$ Triton X-100 and $15 \%$ hydrogen peroxide in $1 \times$ PBS) was added to the test tubes, and the solution was mixed using disposable 10- $\mu$ l loops (BD Difco). Tubes were incubated at room temperature until gas production subsided (approximately 5 to $10 \mathrm{~min}$ ). A standard curve was generated by mixing purified bovine catalase (Sigma, $570 \mathrm{U} / \mu \mathrm{l}$ ) diluted in $1 \times$ PBS with $200 \mu \mathrm{l}$ of catalase reaction buffer. At $10 \mathrm{~min}$ of incubation, images of the tubes were taken with an iPad Air (iOS 12.1.4), and the height from the bottom of the tube to the top of the foam was measured in both the standards and the samples using ImageJ software and an internal 1-inch reference mark for each picture. GraphPad Prism was used to generate the standard curve and interpolate the sample catalase activity using linear regression. The data are presented as catalase activity (units) normalized to the cell number $\left(\mathrm{OD}_{600}\right)$.

RNA isolation and qRT-PCR. Three biological replicate overnight cultures were diluted to a starting $\mathrm{OD}_{600}$ of 0.040 in $2 \mathrm{ml}$ of LB supplemented with ampicillin and IPTG and grown until reaching an $\mathrm{OD}_{600}$ of $\sim 1.0$ at $35^{\circ} \mathrm{C}$ and $220 \mathrm{rpm}$. Next, $1 \mathrm{ml}$ of each replicate was pelleted, and RNA was extracted using the TRIzol reagent following the directions in the manual (Thermo Fisher Scientific). Purified DNA was quantified using a NanoDrop spectrophotometer (Thermo Fisher Scientific). Then, $5 \mu \mathrm{g}$ of purified RNA was treated with DNase (Turbo DNase; Thermo Fisher Scientific). cDNA synthesis was carried out using a GoScript reverse transcription kit (Promega). CDNA was diluted 1:30 into molecular-biology-grade water and used as the template in qRT-PCR analyses with SYBR green (Applied Biosystems) as the method of detection. Reactions consisted of $5 \mu \mathrm{l}$ of $2.5 \mu \mathrm{M}$ primer $1,5 \mu \mathrm{l}$ of $2.5 \mu \mathrm{M}$ primer $2,5 \mu \mathrm{l}$ of diluted cDNA template, and $15 \mu \mathrm{l}$ of $2 \times$ SYBR green consisting of deoxynucleoside triphosphates and AmpliTaq Gold DNA polymerase (see Tables 3 and 4 for the primer sequences). Each plate had technical duplicates and biological triplicate samples, as well as no-reverse transcriptase controls, to check for genomic DNA contamination. A StepOnePlus real-time PCR system was used for qRT-PCR with the following thermocycling conditions: $95^{\circ} \mathrm{C}$ for $20 \mathrm{~s}$, followed by 40 cycles of $95^{\circ} \mathrm{C}$ for $2 \mathrm{~s}$ and $60^{\circ} \mathrm{C}$ for $30 \mathrm{~s}$. Melting curves were included to ensure that $\mathrm{PCR}$ products had single amplicons and that primer dimers were absent. Data were analyzed by the $\Delta \Delta C_{T}$ method using gyrA as a housekeeping or reference target.

Luciferase reporter assays. (i) $V$. cholerae reporter assays. Overnight cultures of $V$. cholerae harboring $k a t B$ transcriptional fusions to luciferase in pBBRlux were diluted 1:100 in $1 \mathrm{ml}$ of LB supplemented with ampicillin, chloramphenicol, and IPTG in $1.5-\mathrm{ml}$ microcentrifuge tubes. Then, $200-\mu \mathrm{l}$ portions of cell solution were aliquoted into the wells of black 96-well plates (Costar). The plates were incubated at $35^{\circ} \mathrm{C}$ with shaking at $220 \mathrm{rpm}$ until the $\mathrm{OD}_{600}$ reached $\sim 0.250$, and the luciferase activity was measured using an Envision plate reader (Perkin-Elmer). The luciferase activity (in relative light units [RLU]) was normalized for the cell number by dividing the RLU by the $\mathrm{OD}_{600}$ at the time of the reading (normalized luminescence). For experiments where $\mathrm{H}_{2} \mathrm{O}_{2}$ were added to the cultures, overnight cultures of $V$. cholerae were diluted as described above except that $\mathrm{H}_{2} \mathrm{O}_{2}$ was added to the cultures at a final concentration of $50 \mu \mathrm{M}$ when the $\mathrm{OD}_{600}$ values reached $\sim 0.225$, followed by shaking at $35^{\circ} \mathrm{C}$ for an additional $30 \mathrm{~min}$ before measuring the luciferase and the $\mathrm{OD}_{600}$.

(ii) E. coli DH10b luciferase assays. Overnight cultures of $E$. coli $\mathrm{DH} 10 \mathrm{~b}$ harboring vectors to modulate transcription factor, c-di-GMP production, and the luciferase reporter were diluted 1:100 as described above and grown at $35^{\circ} \mathrm{C}$ and $220 \mathrm{rpm}$ until the $\mathrm{OD}_{600}$ reached 0.450 . The luciferase activity was measured and normalized to the $\mathrm{OD}_{600}$ to yield the normalized luminescence.

Protein purification and EMSAs. C-terminal His-tagged VpsT purification and electrophoretic mobility shift assay (EMSA) experiments using the FAM-labeled katB promoter region from katB2 were 
TABLE 4 Oligonucleotides used for vector construction

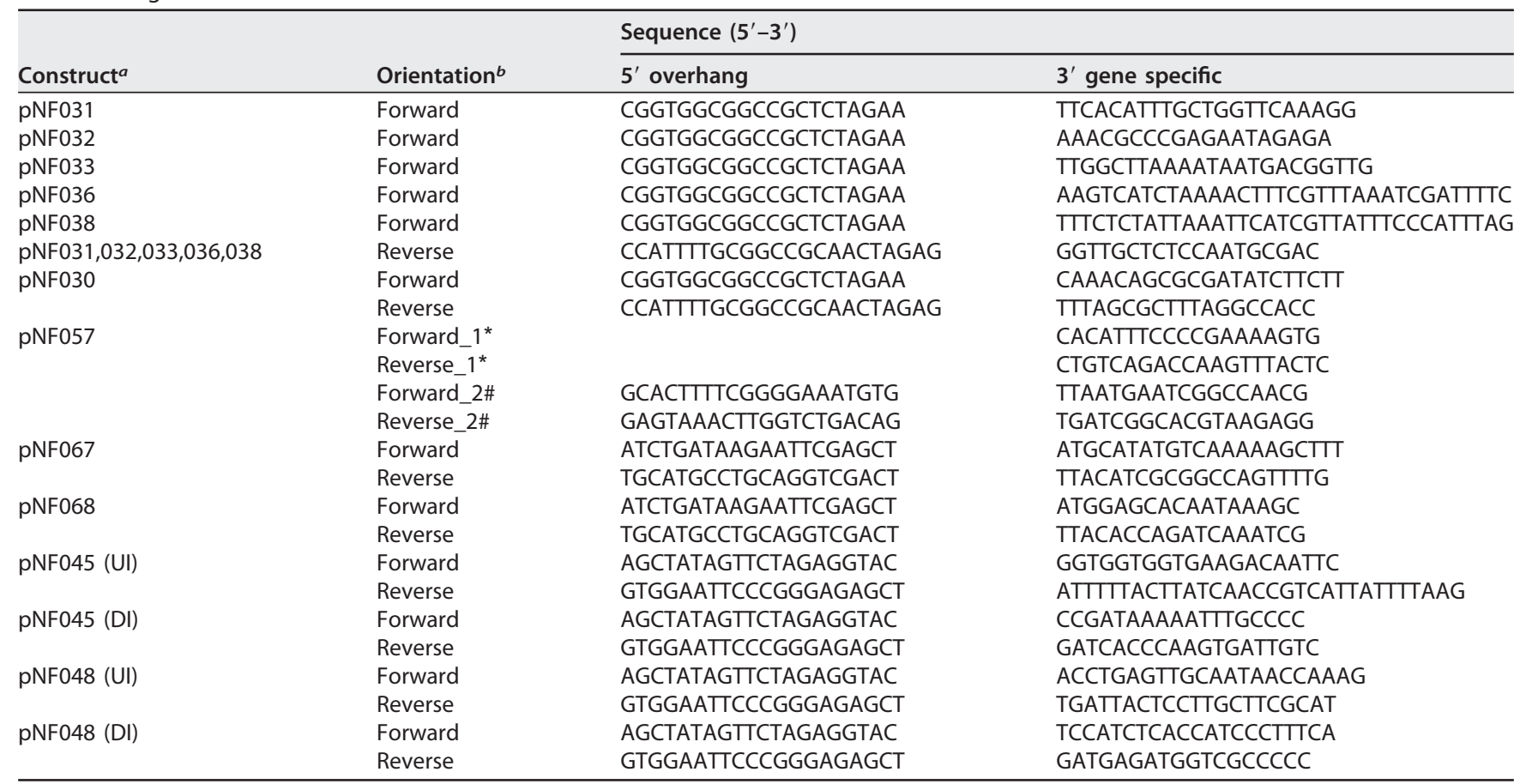

aUl, upstream insert; DI, downstream insert.

$b^{*}$, Forward_1 and Reverse_1, primers used for inverse PCR to amplify pHERD20T backbone without the ampicillin resistance gene; \#, Forward_2 and Reverse_2, primers used to amplify the chloramphenicol acetyltransferase from pBBRlux.

carried out as previously described (21). For purification, an overnight culture of E. coli BL21 harboring the pET28b-VpsT expression construct was diluted 1:100 into $250 \mathrm{ml}$ of LB supplemented with kanamycin in a 1-liter flask. The culture was grown to an $\mathrm{OD}_{600}$ of $\sim 0.7$, at which point $1 \mathrm{mM}$ IPTG was added, and the culture conditions were shifted to $16^{\circ} \mathrm{C}$ with shaking at $160 \mathrm{rpm}$ for $16 \mathrm{~h}$ to induce protein production. Protein purification was carried out by standard Ni-NTA resin purification protocols. For EMSAs, various concentrations of purified His-tagged VpsT (0 to $600 \mathrm{nM}$ ) were incubated with $2.5 \mathrm{nM}$ FAM-labeled katB probe, along with the nonspecific DNA competitor poly(dl-dC) at $1 \mu \mathrm{g} / \mu \mathrm{l}$ in $\mathrm{VpsT}$ buffer ( $25 \mathrm{mM}$ Tris-Cl, $150 \mathrm{mM} \mathrm{NaCl}, 5 \mathrm{mM} \beta$-mercaptoethanol [pH 7.5]) at $30^{\circ} \mathrm{C}$ for $30 \mathrm{~min}$. The binding reaction was loaded into prerun $5 \%$ nondenaturing Tris-borate-EDTA gels, and gel electrophoresis was performed by applying $90 \mathrm{~V}$ for $90 \mathrm{~min}$ at $4^{\circ} \mathrm{C}$. Images of the gels were taken using a Typhoon FLA 9000 imager using settings for FAM label detection and the requisite software (GE Healthcare Life Sciences).

Hydrogen peroxide survival assay. Overnight cultures were diluted to a starting $\mathrm{OD}_{600}$ of 0.040 in $1 \mathrm{ml}$ of LB supplemented with the necessary antibiotics and IPTG in 1.5-ml microcentrifuge tubes. Then, $140-\mu \mathrm{l}$ aliquots were added to a 96-well plate (Costar) and grown to an $\mathrm{OD}_{600}$ of 0.300 . $\mathrm{H}_{2} \mathrm{O}_{2}$ solutions were made from fresh $\mathrm{H}_{2} \mathrm{O}_{2}$ stocks in light-impermeable microcentrifuge tubes and sterile $1 \times$ PBS. At time zero, $10 \mu \mathrm{l}$ of $\mathrm{H}_{2} \mathrm{O}_{2}$ was added to the cell solution, and growth was monitored over time by measuring the $\mathrm{OD}_{600}$.

Measurement of intracellular c-di-GMP. Overnight cultures of $\Delta v p s L$ mutant harboring pBRP1 (QrgBMut) were diluted 1:100 in $2 \mathrm{ml}$ of LB ampicillin in 18-by-150-mm borosilicate test tubes and grown to an $\mathrm{OD}_{600}$ of 1.0. The cultures were split into two 1-ml aliquots in microcentrifuge tubes, and $\mathrm{H}_{2} \mathrm{O}_{2}$ was added to one aliquot at a final concentration of $500 \mu \mathrm{M}$. An equal volume of water was added to the other aliquot as the untreated control. Cultures were incubated statically at room temperature for $30 \mathrm{~min}$ and then collected for total protein quantification and nucleotide extraction. Briefly, $100 \mu$ l of culture was removed from each tube to quantify total protein, pelleted by centrifugation at full speed $(15,000 \times g)$ for $1 \mathrm{~min}$, resuspended in $100 \mu \mathrm{l} 1 \times \mathrm{PBS}$ with $10 \%$ sodium dodecyl sulfate, and boiled at $95^{\circ} \mathrm{C}$ for $10 \mathrm{~min}$. Lysed cell solutions were centrifuged at $15,000 \times g$ for $1 \mathrm{~min}$, and the supernatant was removed and placed in new tubes. Total protein was quantified by using a DC protein assay (Bio-Rad) following the instructions of the manufacturer. Protein standards consisting of bovine serum albumin (provided with the DC protein assay) were used to generate a standard curve to interpolate sample concentrations. Nucleotide extractions were carried out following the protocol as described in reference 38 with the following changes. Portions $(900 \mu \mathrm{l}$ ) of the remaining culture were pelleted at $15,000 \times g$ for $1 \mathrm{~min}$ in a benchtop microcentrifuge. The supernatants were removed, and the remaining pellets were resuspended in $100 \mu \mathrm{l}$ of nucleotide extraction buffer (methanol-acetonitrile-water [40:40:20] with $0.1 \mathrm{~N}$ formic acid). The extraction solution was incubated at $20^{\circ} \mathrm{C}$ for $20 \mathrm{~min}$ and pelleted for $10 \mathrm{~min}$ at $15,000 \times g$. The supernatants were placed into new microcentrifuge tubes, and the solutions were dried overnight using a heated, vacuum centrifuge (SpeedVac concentrator; Savant). The resulting dried pellets 
were resuspended in $100 \mu \mathrm{l}$ of high-pressure liquid chromatography-grade water and subjected to mass spectrometry analysis to quantify the c-di-GMP (39). The data are represented as picomoles of c-di-GMP normalized by total cellular protein $(\mathrm{mg})$.

Statistical analysis. Data are represented as means \pm the standard deviations. Statistical analyses (see details in the figure legends) were calculated using Prism v6 (GraphPad, San Diego, CA). A $P$ value of $<0.05$ was considered statistically significant.

\section{SUPPLEMENTAL MATERIAL}

Supplemental material for this article may be found at https://doi.org/10.1128/AEM .01043-19.

SUPPLEMENTAL FILE 1, PDF file, $0.2 \mathrm{MB}$.

\section{ACKNOWLEDGMENTS}

This material was supported by National Institutes of Health grants GM109259, GM110444, and AI130554 awarded to C.W.M. We thank the College of Natural Science and the Department of Microbiology and Molecular Genetics at Michigan State University for a University Enrichment Fellowship, a Rudolph Hugh Award, and a Bertina Wentworth Fellowship awarded to N.L.F.

We thank Geoffrey B. Severin and Brian Y. Hsueh for critically reading and providing comments on the manuscript.

\section{REFERENCES}

1. Lutz C, Erken M, Noorian P, Sun S, McDougald D. 2013. Environmental reservoirs and mechanisms of persistence of Vibrio cholerae. Front Microbiol 4:375

2. Cooper WJ, Zika RG, Petasne RG, Plane J. 1988. Photochemical formation of hydrogen peroxide in natural waters exposed to sunlight. Environ Sci Technol 22:1156-1160. https://doi.org/10.1021/es00175a004.

3. Glippa O, Engström-Öst J, Kanerva M, Rein A, Vuori K. 2018. Oxidative stress and antioxidant defense responses in Acartia copepods in relation to environmental factors. PLoS One 13:e0195981. https://doi.org/10 .1371/journal.pone.0195981.

4. Diaz JM, Plummer S. 2018. Production of extracellular reactive oxygen species by phytoplankton: past and future directions. J Plankton Res 40:655-666.

5. Wang H, Naseer N, Chen Y, Zhu AY, Kuai X, Galagedera N, Liu Z, Zhu J. 2017. OxyR2 modulates OxyR1 activity and Vibrio cholerae oxidative stress response. Infect Immun 85:e00929-16.

6. Xia X, Larios-Valencia J, Liu Z, Xiang F, Kan B, Wang H, Zhu J. 2017. OxyR-activated expression of Dps is important for Vibrio cholerae oxidative stress resistance and pathogenesis. PLoS One 12:e0171201. https:// doi.org/10.1371/journal.pone.0171201.

7. Heidelberg JF, Eisen JA, Nelson WC, Clayton RA, Gwinn ML, Dodson RJ, Haft DH, Hickey EK, Peterson JD, Umayam L, Gill SR, Nelson KE, Read TD, Tettelin H, Richardson D, Ermolaeva MD, Vamathevan J, Bass S, Qin $H_{\text {, }}$ Dragoi I, Sellers $\mathrm{P}, \mathrm{McD}$ onald L, Utterback T, Fleishmann RD, Nierman WC, White O, Salzberg SL, Smith HO, Colwell RR, Mekalanos JJ, Venter JC, Fraser CM. 2000. DNA sequence of both chromosomes of the cholera pathogen Vibrio cholerae. Nature 406:477-483. https://doi.org/10.1038/ 35020000.

8. Wai SN, Mizunoe Y, Takade A, Kawabata S-I, Yoshida S-I. 1998. Vibrio cholerae $\mathrm{O} 1$ strain TSI-4 produces the exopolysaccharide materials that determine colony morphology, stress resistance, and biofilm formation. Appl Environ Microbiol 64:3648-3655.

9. Chen L-H, Köseoğlu VK, Güvener ZT, Myers-Morales T, Reed JM, D'Orazio SEF, Miller KW, Gomelsky M. 2014. Cyclic di-GMP-dependent signaling pathways in the pathogenic firmicute Listeria monocytogenes. PLoS Pathog 10:e1004301. https://doi.org/10.1371/journal.ppat.1004301.

10. Wang $H$, Xing X, Wang J, Pang B, Liu M, Larios-Valencia J, Liu T, Liu G, Xie S, Hao G, Liu Z, Kan B, Zhu J. 2018. Hypermutation-induced in vivo oxidative stress resistance enhances Vibrio cholerae host adaptation. PLoS Pathog 14:e1007413. https://doi.org/10.1371/journal.ppat.1007413.

11. Romling U, Galperin MY, Gomelsky M. 2013. Cyclic di-GMP: the first 25 years of a universal bacterial second messenger. Microbiol Mol Biol Rev 77:1-52. https://doi.org/10.1128/MMBR.00043-12.

12. Yildiz FH, Dolganov NA, Schoolnik GK. 2001. VpsR, a member of the response regulators of the two-component regulatory systems, is required for expression of vps biosynthesis genes and EPS(ETr)-associated phenotypes in Vibrio cholerae O1 El Tor. J Bacteriol 183:1716-1726. https://doi.org/10.1128/JB.183.5.1716-1726.2001.

13. Krasteva PV, Fong JCN, Shikuma NJ, Beyhan S, Navarro M, Yildiz FH, Sondermann H. 2010. Vibrio cholerae VpsT regulates matrix production and motility by directly sensing cyclic di-GMP. Science 327:866-868. https://doi.org/10.1126/science.1181185.

14. Srivastava D, Hsieh M-L, Khataokar A, Neiditch MB, Waters CM. 2013. Cyclic di-GMP inhibits Vibrio cholerae motility by repressing induction of transcription and inducing extracellular polysaccharide production: FlrA is a c-di-GMP binding transcription factor. Mol Microbiol 90:1262-1276. https://doi.org/10.1111/mmi.12432.

15. Hsieh M-L, Hinton DM, Waters CM. 2018. VpsR and cyclic di-GMP together drive transcription initiation to activate biofilm formation in Vibrio cholerae. Nucleic Acids Res 46:8876-8887. https://doi.org/10.1093/nar/gky606.

16. Kariisa AT, Grube A, Tamayo R. 2015. Two nucleotide second messengers regulate the production of the Vibrio cholerae colonization factor GbpA. BMC Microbiol 15:166. https://doi.org/10.1186/s12866-015-0506-5.

17. Pursley BR, Maiden MM, Hsieh M-L, Fernandez NL, Severin GB, Waters CM. 2018. Cyclic di-GMP regulates TfoY in Vibrio cholerae to control motility by both transcriptional and posttranscriptional mechanisms. J Bacteriol 200:e00578-17. https://doi.org/10.1128/JB.00578-17.

18. Sloup RE, Konal AE, Severin GB, Korir ML, Bagdasarian MM, Bagdasarian M, Waters CM. 2017. Cyclic di-GMP and VpsR induce the expression of type II secretion in Vibrio cholerae. J Bacteriol 199:e00106-17.

19. Metzger LC, Stutzmann S, Scrignari T, Van der Henst C, Matthey N, Blokesch M. 2016. Independent regulation of type VI secretion in Vibrio cholerae by TfoX and TfoY. Cell Rep 15:951-958. https://doi.org/10.1016/ j.celrep.2016.03.092.

20. Wang H, Ayala JC, Benitez JA, Silva AJ. 2014. The LuxR-type regulator VpsT negatively controls the transcription of $r p o S$, encoding the general stress response regulator, in Vibrio cholerae biofilms. J Bacteriol 196: 1020-1030. https://doi.org/10.1128/JB.00993-13.

21. Fernandez NL, Srivastava D, Ngouajio AL, Waters CM. 2018. Cyclic diGMP positively regulates DNA repair in Vibrio cholerae. J Bacteriol 200: e00005-18. https://doi.org/10.1128/JB.00005-18.

22. Mishra S, Imlay J. 2012. Why do bacteria use so many enzymes to scavenge hydrogen peroxide? Arch Biochem Biophys 525:145-160. https://doi.org/10.1016/j.abb.2012.04.014.

23. Waters CM, Lu W, Rabinowitz JD, Bassler BL. 2008. Quorum sensing controls biofilm formation in Vibrio cholerae through modulation of cyclic di-GMP levels and repression of vpsT. J Bacteriol 190:2527-2536. https://doi.org/10.1128/JB.01756-07.

24. Wang $\mathrm{H}$, Chen $\mathrm{S}$, Zhang J, Rothenbacher FP, Jiang $T$, Kan B, Zhong Z, Zhu J. 2012. Catalases promote resistance of oxidative stress in Vibrio cholerae. PLoS One 7:e53383. https://doi.org/10.1371/journal.pone.0053383. 25. Li W, Li M, Hu L, Zhu J, Xie Z, Chen J, He Z-G. 2018. HpoR, a novel 
c-di-GMP effective transcription factor, links the second messenger's regulatory function to the mycobacterial antioxidant defense. Nucleic Acids Res 46:3595-3611. https://doi.org/10.1093/nar/gky146.

26. Srivastava D, Harris RC, Waters CM. 2011. Integration of cyclic di-GMP and quorum sensing in the control of vpsT and aphA in Vibrio cholerae. J Bacteriol 193:6331-6341. https://doi.org/10.1128/JB.05167-11.

27. Zamorano-Sánchez D, Fong JCN, Kilic S, Erill I, Yildiz FH. 2015. Identification and characterization of VpsR and VpsT binding sites in Vibrio cholerae. J Bacteriol 197:1221-1235. https://doi.org/10.1128/JB.02439-14.

28. Elkins JG, Hassett DJ, Stewart PS, Schweizer HP, Mcdermott TR. 1999. Protective role of catalase in Pseudomonas aeruginosa biofilm resistance to hydrogen peroxide. Appl Environ Microbiol 65:4594-4600.

29. Shin D-H, Choi Y-S, Cho Y-H. 2008. Unusual properties of catalase A (KatA) of Pseudomonas aeruginosa PA14 are associated with its biofilm peroxide resistance. J Bacteriol 190:2663-2670. https://doi.org/10.1128/ JB.01580-07.

30. Karatan E, Duncan TR, Watnick PI. 2005. NspS, a predicted polyamine sensor, mediates activation of Vibrio cholerae biofilm formation by norspermidine. J Bacteriol 187:7434-7443. https://doi.org/10.1128/JB.187 .21.7434-7443.2005.

31. Yildiz FH, Schoolnik GK. 1999. Vibrio cholerae O1 El Tor: identification of a gene cluster required for the rugose colony type, exopolysaccharide production, chlorine resistance, and biofilm formation. Proc Natl Acad Sci U S A 96:4028-4033. https://doi.org/10.1073/pnas.96.7.4028.

32. Yildiz FH, Liu XS, Heydorn A, Schoolnik GK. 2004. Molecular analysis of rugosity in a Vibrio cholerae $\mathrm{O} 1 \mathrm{El}$ Tor phase variant: regulation of rugosity. Mol Microbiol 53:497-515. https://doi.org/10.1111/j.1365-2958 .2004.04154.x.

33. Beyhan S, Tischler AD, Camilli A, Yildiz FH. 2006. Transcriptome and phenotypic responses of Vibrio cholerae to increased cyclic di-GMP level.
J Bacteriol 188:3600-3613. https://doi.org/10.1128/JB.188.10.3600-3613 .2006.

34. Ayala JC, Wang H, Silva AJ, Benitez JA. 2015. Repression by H-NS of genes required for the biosynthesis of the Vibrio cholerae biofilm matrix is modulated by the second messenger cyclic diguanylic acid: regulation of biofilm formation by H-NS. Mol Microbiol 97:630-645. https://doi.org/ $10.1111 / \mathrm{mmi} .13058$.

35. Thelin KH, Taylor RK. 1996. Toxin-coregulated pilus, but not mannosesensitive hemagglutinin, is required for colonization by Vibrio cholerae O1 El Tor biotype and 0139 strains. Infect Immun 64:4.

36. Qiu D, Damron FH, Mima T, Schweizer HP, Yu HD. 2008. $P_{B A D}$-based shuttle vectors for functional analysis of toxic and highly regulated genes in Pseudomonas and Burkholderia spp. and other bacteria. Appl Environ Microbiol 74:7422-7426. https://doi.org/10.1128/AEM.01369-08.

37. Iwase T, Tajima A, Sugimoto S, Okuda K, Hironaka I, Kamata Y, Takada K, Mizunoe Y. 2013. A simple assay for measuring catalase activity: a visual approach. Sci Rep 3:3081. https://doi.org/10.1038/srep03081.

38. Fernandez N, Waters CM. 2019. Analyzing diguanylate cyclase activity in vivo using a heterologous Escherichia coli host. Curr Protoc Microbiol 52:e74. https://doi.org/10.1002/cpmc.74.

39. Massie JP, Reynolds EL, Koestler BJ, Cong J-P, Agostoni M, Waters CM. 2012. Quantification of high-specificity cyclic diguanylate signaling. Proc Natl Acad Sci U S A 109:12746-12751. https://doi.org/10.1073/pnas .1115663109 .

40. Dunn AK, Millikan DS, Adin DM, Bose JL, Stabb EV. 2006. New rfp- and pES213-derived tools for analyzing symbiotic Vibrio fischeri reveal patterns of infection and lux expression in situ. Appl Environ Microbiol 72:802-810. https://doi.org/10.1128/AEM.72.1.802-810.2006.

41. Skorupski K, Taylor RK. 1996. Positive selection vectors for allelic exchange. Gene 169:47-52. https://doi.org/10.1016/0378-1119(95)00793-8. 